\title{
Frontiers in Ecology and the Environment
}

\section{Lightweight unmanned aerial vehicles will revolutionize spatial ecology}

Karen Anderson and Kevin J Gaston

Front Ecol Environ 2013; doi:10.1890/120150

This article is citable (as shown above) and is released from embargo once it is posted to the Frontiers e-View site (www.frontiersinecology.org).

Please note: This article was downloaded from Frontiers e-View, a service that publishes fully edited and formatted manuscripts before they appear in print in Frontiers in Ecology and the Environment. Readers are strongly advised to check the final print version in case any changes have been made. 


\title{
Lightweight unmanned aerial vehicles will revolutionize spatial ecology
}

\author{
Karen Anderson* and Kevin J Gaston
}

Ecologists require spatially explicit data to relate structure to function. To date, heavy reliance has been placed on obtaining such data from remote-sensing instruments mounted on spacecraft or manned aircraft, although the spatial and temporal resolutions of the data are often not suited to local-scale ecological investigations. Recent technological innovations have led to an upsurge in the availability of unmanned aerial vehicles (UAVs) - aircraft remotely operated from the ground - and there are now many lightweight UAVs on offer at reasonable costs. Flying low and slow, UAVs offer ecologists new opportunities for scale-appropriate measurements of ecological phenomena. Equipped with capable sensors, UAVs can deliver fine spatial resolution data at temporal resolutions defined by the end-user. Recent innovations in UAV platform design have been accompanied by improvements in navigation and the miniaturization of measurement technologies, allowing the study of individual organisms and their spatiotemporal dynamics at close range.

Front Ecol Environ 2013; doi:10.1890/120150

$\mathbf{R}$ emote-sensing techniques have transformed ecological research by providing both spatial and temporal perspectives on ecological phenomena that would otherwise be difficult to study (eg Kerr and Ostrovsky 2003; Running et al. 2004; Vierling et al. 2008). In particular, a strong focus has been placed on the use of data obtained from spaceborne remote-sensing instruments because these provide regional- to global-scale observations and repeat timeseries sampling of ecological indicators (eg Gould 2000). The main limitation of most of the research-focused satellite missions is the mismatch between the pixel resolution of many regional-extent sensors (eg Landsat [spatial resolution of $\sim 30 \mathrm{~m}$ ] to Moderate Resolution Imaging Spectroradiometer [spatial resolution of $\sim 1 \mathrm{~km}$ ]), the revisit period (eg 18 days for Landsat), and the scale of many ecological processes. Indeed, data provided by these platforms are often "too general to meet regional or local objectives" in ecology (Wulder et al. 2004). To address this limitation, a

\section{In a nutshell:}

- Ecologists require data collected at appropriate spatial and temporal resolutions for a range of studies

- Unmanned aerial vehicles (UAVs) can carry various imaging or non-imaging payloads to provide spatial datasets for a variety of end-users

- The benefits of UAVs are that (1) survey revisit periods (ie the number of repeat flights over a designated site) can be user controlled, (2) low-altitude flight allows sensors to observe the ground from more proximal positions and potentially collect finer spatial resolution data, and (3) operating costs are low

- We review current approaches to UAV deployment, summarize their demonstrated and potential applications, and suggest new ways in which UAVs could be used to underpin novel, scale-appropriate environmental science research

Environment and Sustainability Institute, University of Exeter, Penryn, Cornwall, UK* (karen.anderson@exeter.ac.uk) range of new (largely commercially operated) satellite sensors have become operational over the past decade, offering data at finer than $10-\mathrm{m}$ spatial resolution with more responsive capabilities (eg Quickbird, IKONOS, GeoEye1, OrbView-3, WorldView-2). Such data are useful for ecological studies (Fretwell et al. 2012), but there remain three operational constraints: (1) a high cost per scene; (2) suitable repeat times are often only possible if oblique view angles are used, distorting geometric and radiometric pixel properties; and (3) cloud contamination, which can obscure features of interest (Loarie et al. 2007). Imaging sensors on board civilian aircraft platforms may also be used; these can provide more scale-appropriate data for fine-scale ecological studies, including data from light detection and ranging (LiDAR) sensors (Vierling et al. 2008). In theory, these surveys can be made on demand, but in practice data acquisition is costly, meaning that regular time-series monitoring is operationally constrained.

A new method for fine-scale remote sensing is now emerging that could address all of these operational issues and thus potentially revolutionize spatial ecology and environmental science. Unmanned aerial vehicles (UAVs) are lightweight, low-cost aircraft platforms operated from the ground that can carry imaging or non-imaging payloads. UAVs offer ecologists a promising route to responsive, timely, and cost-effective monitoring of environmental phenomena at spatial and temporal resolutions that are appropriate to the scales of many ecologically relevant variables. Emerging from a military background, there are now a growing number of civilian agencies and organizations that have recognized the possible applications of UAVs, including the National Oceanic and Atmospheric Administration, which states that UAVs "have the potential to efficiently and safely bridge critical information gaps" in data-sparse locations "and advance understanding of key processes in Earth sys- 
tems" (NOAA 2012). Here, we review available UAV systems, discuss the ecological research directions that such platforms could service, and highlight some of the scientific opportunities and challenges that lie ahead.

\section{- Types of UAVs}

There are abundant designs for UAV platforms. The key distinction in terms of their capability and ease of operation is their physical size and power, which limits their payload carrying capacity, operating altitude, and range. Their size and power also defines the applications that can be supported by each class of UAV. Various different classification schemes for UAVs exist (Mackenzie 2009; Watts et al. 2012), but for present purposes we make a simple differentiation into four main types (Table 1), focusing on the principal areas where ecologists have benefited or could benefit from these systems.

\section{Large and medium UAVs}

In the large UAV class, most ecologically relevant research has been undertaken with adapted military-grade platforms, the main example being the National Aeronautics and Space Administration's (NASA's) Ikhana, a modified MQ-9 Predator-B vehicle. Ikhana supports applications where manned aircraft deployment would be potentially unsafe or inefficient, for instance in real-time monitoring of the spread of forest wildfires in the western US (Ambrosia et al. 2010). The main operational constraint in the use of large UAVs such as Ikhana is the financial cost. Acquiring, developing, and deploying these platforms is expensive because ground operations are complex; for example, Ikhana requires a runway for takeoff and landing, and highly specialized ground support staff for every mission. Similar deployment constraints apply to mediumclass UAVs, the primary example being NASA's Sensor Integrated Environmental Remote Research Aircraft (SIERRA) platform (Fladeland 2009). SIERRA has so far been tasked only with low-altitude missions for Earth science investigations (eg tropospheric chemistry, Arctic ice reconnaissance; Fladeland et al. 2011), but there is the potential for it to be used for broader applications, including ecological surveys (Fladeland 2009). In addition, medium and large UAV platforms have high operational costs, rendering them impractical for most ecological research needs. For these reasons, this review will provide a critical overview of smaller, lightweight UAV platforms for ecological science, which have a potentially broader scope.

\section{Small, mini, micro, and nano UAVs}

A plethora of small, mini, and micro UAVs, including off-the-shelf designs and user-built kit systems, are available to the ecological researcher. Most ecological studies to date have been undertaken with these smaller, "low and slow" systems, which typically "weigh less than 20 kilograms, have flight times of a few hours, and have very limited ranges" (Hardin and Jensen 2011). The earliest of these were "hobbyist-grade" model aircraft (Watts et al. 2012), which had varying degrees of success. Subsequent technological development has been driven by recognition of their potential to deliver high-quality spatial data from portable and cost-effective platforms to a range of science end-users (Watts et al. 2012). The expansion of applications has also been facilitated by miniaturization and cost reductions among inertial sensors, global positioning system (GPS) devices, and embedded computers (Berni et al. 2009), and there are now numerous miniaturized sensors suited to UAV deployment. Three types of UAVs exist in this size range: (1) fixed-wing platforms, (2) rotor-based copter systems, and (3) the newest generation of nano-UAVs that are aerodynamically different from other such systems. Each design offers operational advantages (Figure 1) and there are multiple considerations when deploying them in different environments (Panel 1).

\section{Fixed-wing UAVs}

Examples of fixed-wing UAVs include (1) the Small Unmanned Meteorological Observer (SUMO; Figure 1a), a "recoverable radiosonde" UAV designed around a low-cost remote aircraft construction kit (Mayer et al. 2012); (2) the slightly larger Meteorological Mini Aerial Vehicle ( $\mathrm{M}^{2} \mathrm{AV}$; Figure 1b; Martin et al. 2011); (3) the Quest UAV system (Table 1; Carlisle et al. in review), which weighs $2 \mathrm{~kg}$, has a high density foam body with a 2-m wingspan, and can carry a payload of up to $1.5 \mathrm{~kg}$ over a pre-determined GPS-waypoint defined route; and (4) the BAT-3 system (Laliberte and Rango 2009), which weighs $10 \mathrm{~kg}$, has a 1.8 -m wingspan, has a flight time of 2-5 hours, can fly to altitudes of $2700 \mathrm{~m}$, is launched from a vehicle rooftop (Figure 1c), and is configured for video, multispectral camera, and still-camera payloads. Fixed-wing platforms such as these can typically travel up to a few kilometers from the launch site, but aviation rules usually state that there must be a visual line of sight from the operator to the UAV; these rules vary between countries and regulations change frequently, in response to the increasing number of UAV users and operators.

Fixed-wing systems can usually travel at faster speeds than rotor-based systems, although overall speeds are still low, helping to minimize image smear issues when carrying a camera-based payload (Hardin and Jensen 2011). Fixed-wing systems are generally larger in size (with wingspans of 1-3 m) than rotor-based systems, and in most cases launch and landing is performed manually. Launching tends to rely on bungee propulsion, whereas landing is usually accomplished through a controlled glide onto soft ground. Once in the air, navigation and flight planning are enabled through readily available GPS-based autopilot guidance tools, which can navigate the aircraft through a series of pre-defined waypoints or 


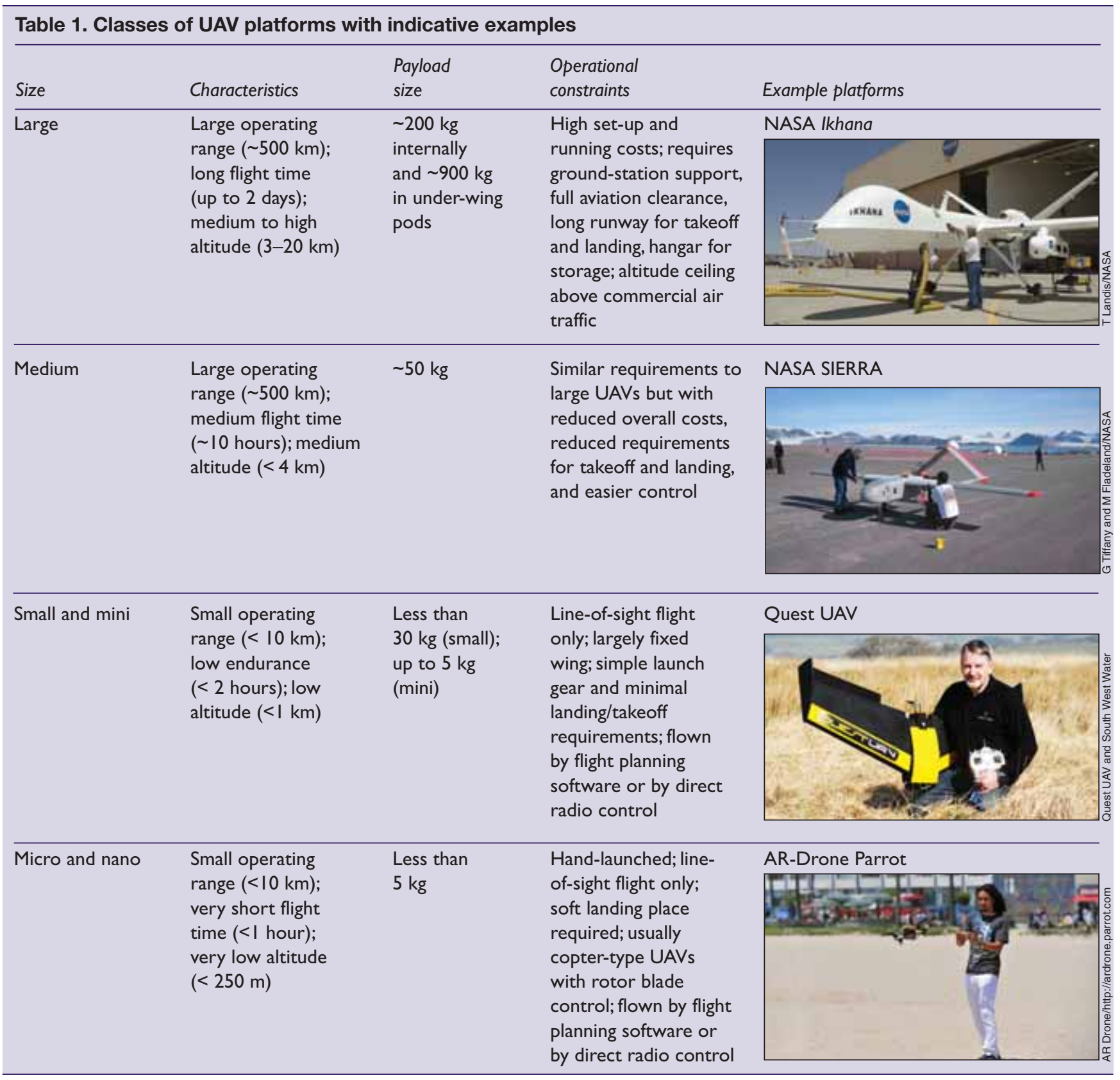

along a pre-determined flight path (Hardin and Jensen 2011). There are a growing number of commercially available, "ready to fly", lightweight fixed-wing systems (eg senseFly, www.sensefly.com; Gatewing, www.gate wing.com). Both are designed to collect geometrically corrected aerial photographs for generating rapid geographic information system (GIS)-ready image mosaics. A notable advantage of these types of UAVs is that the user needs minimal experience to operate the platform, given that flight operations are controlled from a simple interface.

\section{Rotor-based UAVs}

Rotor-based "copter" UAVs differ in capability from the fixed-wing systems in that the former are able to hover over fixed targets, making them suitable for vertical profil- ing experiments as well as spatial surveys. A variety of rotor-based systems are available, ranging from true helicopter systems in the small UAV class (eg the CSIRO Autonomous Helicopter System [Merz and Chapman 2011]; Figure 1d) to more lightweight systems known as "microcopters" (Figure 1). The family of microcopters includes quadcopters or quadrotors (Hendrickx et al. 2011) and octocopters (Israel 2011), which feature four and eight sets of rotor blades, respectively. Two distinct forms of rotor-based UAVs exist: those with blades arranged around a central body, in which the payload is carried (eg Droidworx system; Figure 1f), and those where the rotors are arranged along two arms located on either side of the payload (eg the Ascending Technologies Falcon system; Figure 1e). These UAVs can be controlled in a more precise manner than many other systems because the angular 
velocities of the numerous rotor blades can easily be adjusted. An upsurge in production and application of these systems has been driven by the availability of fast, precise, and affordable accelerometers that do not require complex mechanics for flight stability and ease of control. Wallace et al. (2011) reported that microcopter UAVs exhibit less vibration than fixed-wing systems, making them better suited to photogrammetric data capture. Larger payloads can be transported as more rotors are added, but the size of the payload is still potentially limiting; for example, the Droidworx Microcopter AD-8 (Figure 1f) can carry a 2.8-kg payload (including batteries, an inertial measurement unit, a GPS antenna, and a sensing device). As more rotor blades are added, the risk of system crashes is reduced; octocopters, for instance, can remain airborne even if one of the rotors loses power.

\section{New classes of UAVs}

The development of highly miniaturized UAVs is new, and few civilian examples are described in the literature. Perhaps the most interesting developments have been in the field of "ornithopters" (Mackenzie 2012a), of which the Nano-Hummingbird UAV (Figure $1 \mathrm{~g}$ ) is the lightest. This is a small (19 $\mathrm{g}$ in weight, with a wingspan of 16 $\mathrm{cm})$, low-altitude, and very short duration UAV, designed to carry a miniaturized video camera. It was intended for use in situations where larger vehicles would create a noticeable disturbance (Mackenzie $2012 \mathrm{~b}$ ). Also in the miniature class, the Mirador (Figure $1 i$ ) is a $25-\mathrm{cm}$-long, fixed-wing UAV that is powered by miniature fuel cells and has a 20-minute flight time. There is also a growing class of UAVs that are based on biometric designs. Mackenzie (2012a) reported on two additional platforms, the Smartbird and the Phoenix (Figure $1 \mathrm{~h}$ ), that are larger in size and are in theory more maneuverable than fixed-wing or rotor-based microcopters. These systems are still highly experimental and in development, and before operational use by ecologists can be realized, research efforts are needed to improve their stability optimization (Orlowski and Girard 2012). Focus must also be directed toward developing miniature, lightweight sensors for navigation, stabilization, and measurement (Hermans and Decuypere 2005). Although these systems were not originally designed with environmental research in mind, we suggest that new innovations in the development of miniature UAV platforms could provide valuable data for ecological studies, such as insights into animal behavior in environments where human observers and larger UAVs would otherwise disturb the subject organism.

Beyond the scientific market, the newest and most financially accessible UAV platforms are emerging in the recreational consumer market. One such system is

\section{Panel 1. Operational considerations for lightweight UAVs}

There are many operational considerations to take into account when deploying lightweight UAV systems for spatial research; these fall into four main categories:

\section{(I) Platform constraints}

(a) Restricted payload capacity limits mission endurance (and hence flying time) of lightweight UAVs. This could be counteracted in fixed-wing systems by wing-mounted solar panels that would provide top-up power while the UAV is in flight, following the lead set by NASA's Helios mission.

(b) Gliding to a landing position on variable terrain may cause damage to the platform and attached sensor(s) - thus, platform and payload weight should be kept low.

\section{(2) Sensor constraints}

(a) Limited payload size restricts users to simple sensors with few moving parts, affecting:

(i) Radiometric data quality: reproducibility of data may be compromised in smaller systems with lower signal-to-noise capabilities. Empirical radiometric calibration of data products would ensure long-term data quality (Smith and Milton 1999).

(ii) Geometric stability and vibration effects of the platform may affect data quality. This can be addressed by decoupling the sensor from the platform using a gimbal mount, or treating the sensor and platform as a single unit and post-processing the data using navigation information stored by a UAV attitude system. Similar techniques have already been widely used in airborne remote sensing.

(b) Validating the geometric accuracy of spatial data products should be considered in relation to accurately surveyed ground control points.

(3) Operating constraints

(a) Lightweight UAVs are challenging to pilot, even if GPS-enabled navigation is included (takeoff and landing are typically pilot-controlled).

(b) Flight restrictions imposed by civil and federal aviation laws limit deployment to small ranges and unpopulated areas.

(c) Utility lines pose a potential operational hazard and flying zones should therefore be restricted to areas where these are absent.

\section{(4) Environmental constraints}

(a) There is an increased risk of complete system loss in high winds.

(b) Site conditions, such as dense tree canopy cover, may make real-time tracking difficult or prevent platform retrieval if the UAV goes off track. 

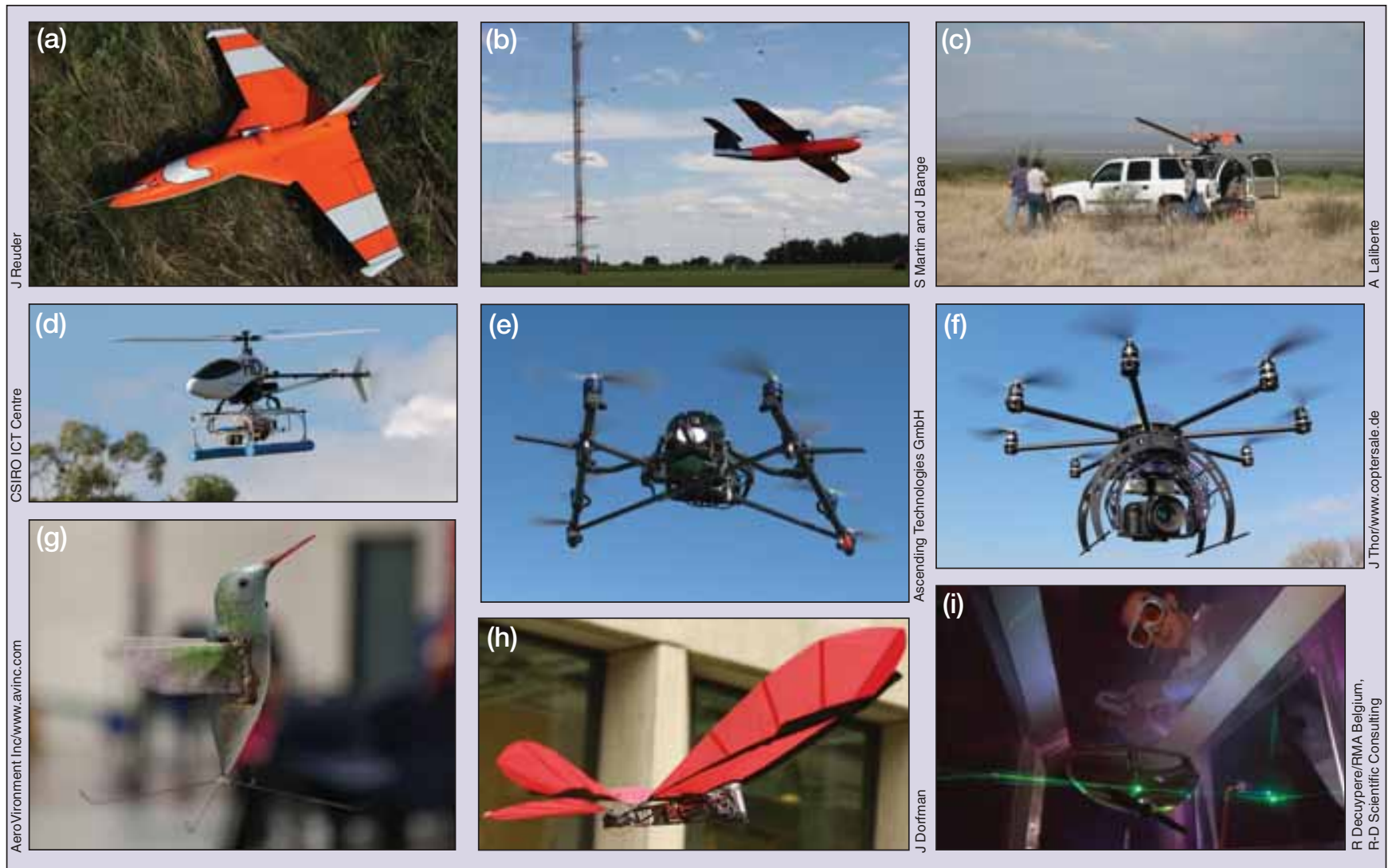

Figure 1. Some examples of the current variety of lightweight $U A V$ s, before, during, and after operation: (a) SUMO, (b) M ${ }^{2} A V$ Carolo, (c) BAT-3 UAV, (d) CSIRO Autonomous Helicopter System, (e) Ascending Technologies Falcon Octocopter, (f) Droidworx Microcopter AD-8, (g) Nano-Hummingbird UAV, (h) Phoenix "ornithopter" UAV, and (i) Mirador micro-UAV.

the AR-Drone "Parrot", the first lightweight quadcopter to be controlled by a smartphone or tablet PC application (Table 1; http://ardrone.parrot.com). This platform, which can be purchased for about US $\$ 400$, can carry a basic forward-viewing video and nadir-viewing (ie a sensor viewing a surface from directly overhead or at 90 degrees to the surface normal) camera system that could easily be applied to spatial ecological research. The main limitation of the Parrot is its short duration; flying time is only about 12 minutes. However, the system can easily be serviced with spare batteries and is repaired from assorted, readily available spare parts. Additionally, there is a large online community of UAV enthusiasts (www.DIYDrones.com) who have collectively developed the multirotor "ArduCopter" platform. This UAV, which is operated through an open-source autopilot system ("ArduPilot-Mega") created by the DIYDrones community, offers GPS-waypoint flying, gyro-stabilized flight, and sonar-guided takeoff and landing capabilities. ArduCopter features on-board flight telemetry and data storage, and includes a camera stabilization apparatus to minimize geometric distortion of data that would otherwise be caused by platform wobble. The open-source nature of the DIYDrones internet community means that ecologists wishing to adapt the ArduCopter for specific applications would have online access to expert guidance and best practices.

\section{Sensor systems for deployment on lightweight UAVs}

Although the focus of this paper is on lightweight UAV platforms, it is necessary to discuss the types of sensors that can currently be deployed on such systems. The main limitation to instrument deployment in this class is weight; most platforms are limited to carrying payloads of less than $3 \mathrm{~kg}$, but some models are capable of only carrying much lighter payloads, which may affect the quality of the data collected (Panel 1). Furthermore, the total payload that can be carried by such platforms must include operational apparatus, such as batteries, cables, data storage equipment, and telemetry systems; in other words, the sensor alone is not the total payload. Most lightweight UAV platforms would therefore be incapable of carrying multisensor systems, so users requiring such data would need to deploy the UAV multiple times over the same site. Combining data from multiple sensors post-flight would necessitate high-quality GPS tracking and tagging of the acquired data at the point of collection, and there would also be a time delay between data acquisitions; datasets thus would not be perfectly matched in time. Lightweight data-recording can be achieved through two main types of system at the moment:

(1) Non-imaging systems that can be triggered remotely or set to record data at regular time intervals during the flight. These could include meteorological sen- 
sors, broadband or narrowband pyranometer-type radiometric sensors, or lightweight miniaturized hyperspectral radiometers (eg those manufactured by Ocean Optics [www.oceanoptics.com]). The motion of the UAV, coupled with precise recording of position at the point of data collection, would allow the user to build up a spatial picture of a variable using geometrically attributed point measurements collected across a spatial extent.

(2) Lightweight imaging systems, including standard RGB cameras, and lightweight thermal systems (eg those manufactured by FLIR [www.flir.com]) have already been tested on UAV platforms and shown to produce useful data for diverse ecological applications (see "Applications" section below)

The main technical challenges associated with both approaches relate to the radiometric and geometric quality of the data. Users need to be mindful of data smear issues caused by the motion of the platform and the importance of capturing accurate information on platform attitude (ie the three-dimensional [3D] information describing the aircraft's roll, pitch, and yaw) for each data point so as to allow angular illumination and measurement characteristics to be understood. This information is also needed to ensure long-term radiometric data quality because image brightness values will vary depending on the angle of illumination and view. Panel 1 provides further details on some of these considerations.

\section{Applications}

Lightweight UAVs have been used in a broad range of ecological research projects, although these represent only a small fraction of the full spectrum of possible applications.

\section{Population ecology}

Both pure and applied population ecology studies commonly require time-series data of abundance and/or distribution. UAVs can provide an effective means of obtaining such information because of the potential for suitable resurvey periods. Jones et al. (2006) experimented with a folding fixed-wing UAV for bird and reptile surveys, and provided a helpful commentary on operational issues for such applications. UAVs have also been used for successful monitoring of black-headed gull (Chroicocephalus ridibundus) colonies (Sarda-Palomera et al. 2012) where GIS approaches were used to assess population sizes via close-range digital imagery. Repeat surveys of the colonies using this method caused minimal disturbance, allowing the temporal and spatial variation in the number of breeding pairs to be monitored. In addition, UAVs may offer advantages as platforms for performing marine ecological surveys, particularly when species are susceptible to disturbance from boat vibrations (eg beaked whales [Ziphiidae]; Koski et al. 2009).

Thermal imaging systems on UAVs show great promise for monitoring the distribution and abundance of organisms. Israel (2011) used a thermal camera on board an octocopter to detect the presence and location of roe deer (Capreolus capreolus) in fields. The primary aim was to develop a system to reduce fawn mortality caused by mowing machinery, but similar methods could be widely applied to general population monitoring of various species (Gill et al. 1997). Night-time thermal imaging from UAVs would provide improved discrimination among individuals because they would be thermally less cryptic in relation to their surroundings. This approach would work well for monitoring nocturnal species or spatiotemporal monitoring of population and behavioral dynamics, although operations may be constrained by regulatory restrictions on night-time flying.

The extraction of useful ecological population data from UAV-acquired imagery has primarily relied on manual interpretation of the spatial datasets produced. This can be time-consuming if imagery is collected over large areas, or if there are numerous individual organisms to count or map. The inclusion of automatic pattern recognition techniques into data-processing procedures can accelerate the process (Abd-Elrahman 2005) and could easily be used with UAV data.

\section{Vegetation dynamics}

The primary advantages of using lightweight UAVs for research into vegetation dynamics is that individual plants can be spatially resolved if flight paths are at sufficiently low altitude (Getzin et al. 2012), revisit times can be optimized to the phenological cycle of target species, and UAVs are able to carry miniature narrowband or hyperspectral radiometers or thermal cameras to capture patterns in biophysical variables. Getzin et al. (2012) demonstrated how the fine spatial resolution provided by UAV photography ( $7-\mathrm{cm}$ pixel size) is useful in providing scale-appropriate data for describing canopy-gap metrics relating to the floristic biodiversity of a forest understory. Herwitz et al. (2004) also commented on the benefits of using the maneuvering capability of UAVs to avoid contamination of image data by clouds. There are financial incentives too; Hardin and Jackson (2005) used a UAVmounted, 35-mm single-lens reflex (SLR) camera to overcome the high costs of repeated aircraft surveys over rangelands of the mid-western US, negating the need for time-consuming field transects. Laliberte and Rango (2009) have further expanded this approach by using textural image processing approaches to differentiate ecological communities in rangelands.

Precision agriculture (ie where farmers can adjust agricultural treatments at fine scales according to local conditions) is particularly well positioned to benefit from UAV innovations (Lelong et al. 2008) because fine spatial resolution data with quick turnaround times are required for 
accurate assessment of crop productivity (Berni et al. 2009; Guillen-Climent et al. 2012; Zarco-Tejada et al. 2012). Thermal imaging from UAVs can provide a useful indicator of the water status of vegetation; Berni et al. (2009) used thermal images acquired through a UAV platform to characterize the thermal properties of crop canopies. In combination with narrowband optical products (ie where optical data are targeted at specific regions of the electromagnetic spectrum to highlight specific biogeochemical processes) collected simultaneously, spatially validated estimates of water stress were obtained. Similarly, Zarco-Tejada et al. (2012) used thermal data in combination with fluorescence spectroscopy obtained from a UAV to measure water stress in an orchard canopy. Guillen-Climent et al. (2012) further adapted these methods to look at leaf area index and fraction of absorbed photosynthetically active radiation retrievals from a multispectral camera mounted on a UAV.

An emerging opportunity is the use of fleets of simultaneously deployed "swarming" UAVs. Although this model for UAV use has not yet been realized in agriculture, forest managers are moving toward using fleets of UAVs for near-real-time fire reporting (Merino et al. 2012), because a coordinated group of UAVs allows the spatial range limitations of a single platform to be overcome. Similar techniques are already being tested in unmanned underwater monitoring systems (eg www. roboshoal.com).

Several potential growth areas are evident in studies of vegetation dynamics with UAVs. First, Hunt et al. (2011) demonstrated how standard, visible-range cameras can be modified to measure near infrared light, meaning that spatial vegetation indices can be easily measured from UAVs. Second, lightweight miniaturized LiDAR sensors are in development (Lin 2011) with UAV deployment in mind. A third possibility is evidenced by studies showing how structural point cloud data (ie $\mathrm{x}, \mathrm{y}, \mathrm{z}$ data revealing the 3D geographical position of landscape objects) can be extracted from overlapping photographic images of landscapes. This technique was originally envisioned from ground-based imaging positions, but recently published articles suggest that UAV platforms could provide an efficient means of generating "structure from motion" data from synoptic perspectives (Dandois and Ellis 2010; Rosnell and Honkavaara 2012). Commercially available systems (eg Gatewing, senseFly) already offer automated solutions to modeling landscape geometry from overlapping images. Results from scientific studies confirm that such approaches are further able to support more advanced, low-cost, 3D remote sensing of vegetation structure (Figure 2). Wallace et al. (2012) illustrated how these approaches could also be applied to tree-crown structural mapping from an octocopter, demonstrating that within-canopy structural information can be determined. All of these approaches have applications in a range of ecological and environmental disciplines because canopy structure is often linked to underlying abiotic drivers (eg hydrology) and is an indicator of other key variables (eg microclimate, biodiversity).

\section{Ecosystem processes}

Meteorological scientists have long recognized the potential for assimilating data obtained from UAV platforms into their research; for instance, a study by Jonsson et al. (1980) was one of the first to use a remote-controlled aircraft (SUMO) for vertical temperature profiling. Equipped with a suite of miniature sensors, SUMO (Figure 1a) has successfully collected temperature, relative humidity, and wind profiles at altitudes as high as 3 $\mathrm{km}$ (Mayer et al. 2012). Similarly, the $\mathrm{M}^{2} \mathrm{AV}$ (Figure 1b) has been used to reveal the turbulent structure of the atmospheric boundary layer to $1500 \mathrm{~m}$ above the ground (Martin et al. 2011; van den Kroonenberg et al. 2012). In the case of fixed-wing UAVs, an upward circling flight pattern through the lower atmosphere is used. We suggest that the newer rotor-based systems could be more effective for addressing atmospheric profiling questions because they can hover at different elevations. UAVs undoubtedly offer advantages over other methods (eg balloons, radiosondes, tall towers) for atmospheric profiling 
because they can provide information on the temporal and spatial heterogeneities of the boundary layer, in both horizontal and vertical domains. Furthermore the flexibility, comparative low cost, and ease of UAV deployment overcome the limitations of the temporal "snapshot" that would otherwise be gathered by manned aircraft, allowing for a more thorough understanding of atmospheric characteristics. Moreover, unlike atmospheric balloons, UAVs are recoverable and reusable. In addition, vibration is much reduced in UAVs as compared with airborne vehicles powered by combustion engines, resulting in potentially greater precision in meteorological measurements (Martin et al. 2011).

The relevance of this work to ecology and environmental science is straightforward. For example, many studies that quantify land-surface atmosphere fluxes of gases or aerosols at eddy covariance towers (Baldocchi et al. 2001) require an understanding of meteorological conditions over the spatial extent of the flux footprint through time; fine-scale monitoring by UAVs within the footprint could allow fluxes to be up-scaled to satellite observations. Current techniques for up-scaling rely on ground-based measurements of biophysical canopy variables (Balzarolo et al. 2011) or "phenocam" observations from SLR cameras (Migliavacca et al. 2011). UAVs could be adapted to deploy these technologies and as such represent a potentially very powerful tool for ecological scaling studies. Another area of research in which UAVs may be effective is the study of insect population dynamics (Carvell et al. 2011), which requires finescale spatiotemporal data describing boundary-layer meteorology to better understand insect behavior. UAVs could provide essential data for recognizing the link between the spatiotemporal distribution of abiotic variables and corresponding biotic responses.

\section{Conclusions}

The field of spatial ecology is severely hampered by the difficulties of obtaining appropriate data, and particularly data at fine spatial and temporal resolutions and over prolonged periods of time, at reasonable costs. The use of UAVs is a major step toward more effective and efficient operational monitoring and management of natural resources. In flying low and slow, and being comparatively affordable, UAVs offer scientists new opportunities for scale-appropriate measurement of ecological phenomena, delivering fine spatial resolution data at user-controlled revisit periods. Developments in the navigational capabilities and ongoing miniaturization of measurement technologies (ie smaller, lighter payloads) will enable data to be obtained that, until now, ecologists have only dreamed of collecting.

\section{Acknowledgements}

We thank SL Chown and EJ Milton for comments and discussion. KJG is supported by the European Research Council under the European Union's Seventh
Framework Programme (FP7/2007-2013)/ERC grant agreement \#268504.

\section{References}

Abd-Elrahman A. 2005. Development of pattern recognition algorithm for automatic bird detection from unmanned aerial vehicle imagery. Surv Land Inf Sci 65: 37-45.

Ambrosia VG, Wegener S, Zajkowski T, et al. 2010. The Ikhana unmanned airborne system (UAS) western states fire imaging missions: from concept to reality (2006-2010). Geocarto Int J 26: 85-101.

Baldocchi D, Falge E, Gu LH, et al. 2001. FLUXNET: a new tool to study the temporal and spatial variability of ecosystem-scale carbon dioxide, water vapor, and energy flux densities. B Am Meteorol Soc 82: 2415-34.

Balzarolo M, Anderson K, Nichol C, et al. 2011. Ground-based optical measurements at European flux sites: a review of methods, instruments and current controversies. Sensors 11: 7954-81.

Berni JAJ, Zarco-Tejada PJ, Suarez L, et al. 2009. Thermal and narrowband multispectral remote sensing for vegetation monitoring from an unmanned aerial vehicle. IEEE T Geosci Remote 47: $722-38$.

Carlisle B, Dunning SA, Lim M, et al. Accurate, highly automated ortho-photo and DSM production from UAV imagery. Remote Sens Environ. In review.

Carvell C, Osborne JL, Bourke AFG, et al. 2011. Bumble bee species' responses to a targeted conservation measure depend on landscape context and habitat quality. Ecol Appl 21: 1760-71.

Dandois JP and Ellis EC. 2010. Remote sensing of vegetation structure using computer vision. Remote Sensing 2: 1157-76.

Fladeland M. 2009. SIERRA: a new unmanned aircraft for Earth science investigations. Moffett Field, CA: NASA Ames Research Center. www.fuentek.com/downloads/AR-0022\%20SIERRATOP.pdf. Viewed 16 Apr 2012.

Fladeland M, Sumich M, Lobitz B, et al. 2011. The NASA SIERRA science demonstration programme and the role of small-medium unmanned aircraft for Earth science investigations. Geocarto Int J 26: 157-63.

Fretwell PT, LaRue MA, Morin P, et al. 2012. An emperor penguin population estimate: the first global, synoptic survey of a species from space. PLoS ONE 7: e33751.

Getzin S, Wiegand K, and Schöning I. 2012. Assessing biodiversity in forests using very high-resolution images and unmanned aerial vehicles. Method Ecol Evol 3: 397-404.

Gill RMA, Thomas ML, and Stocker D. 1997. The use of portable thermal imaging for estimating deer population density in forest habitats. J Appl Ecol 34: 1273-86.

Gould W. 2000. Remote sensing of vegetation, plant species richness, and regional biodiversity hotspots. Ecol Appl 10: 1861-70.

Guillen-Climent M, Zarco-Tejada PJ, Berni J, et al. 2012. Mapping radiation interception in row-structured orchards using 3D simulation and high-resolution airborne imagery acquired from a UAV. Precis Agric 13: 473-500.

Hardin PJ and Jackson MW. 2005. An unmanned aerial vehicle for rangeland photography. Rangeland Ecol Manag 58: 439-42.

Hardin PJ and Jensen RR. 2011. Small-scale unmanned aerial vehicles in environmental remote sensing: challenges and opportunities. GISci Remote Sens 48: 99-111.

Hendrickx M, Gheyle W, Bonne J, et al. 2011. The use of stereoscopic images taken from a microdrone for the documentation of heritage - an example from the Tuekta burial mounds in the Russian Altay. J Archaeol Sci 38: 2968-78.

Hermans D and Decuypere R. 2005. A challenge for micro and mini UAV: the sensor problem. www.dtic.mil/cgi-bin/ GetTRDoc?AD=ADA471957. Viewed 27 May 2012.

Herwitz SR, Johnson LF, Dunagan SE, et al. 2004. Imaging from an 
unmanned aerial vehicle: agricultural surveillance and decision support. Comput Electron Agr 44: 49-61.

Hunt E, Hively W, McCarty G, et al. 2011. NIR-green-blue highresolution digital images for assessment of winter cover crop biomass. GISci Remote Sens 48: 86-98.

Israel M. 2011. A UAV-based roe deer fawn detection system. www.isprs.org/proceedings/XXXVIII/1-C22/papers/israel.pdf. Viewed 27 May 2012.

Jones GP, Pearlstine LG, and Percival HF. 2006. An assessment of small unmanned aerial vehicles for wildlife research. Wildlife Soc B 34: 750-58.

Jonsson I, Mattson JO, Okla L, et al. 1980. Photography and temperature measurements from a remotely piloted vehicle. Oikos 35: $120-25$.

Kerr JT and Ostrovsky M. 2003. From space to species: ecological applications for remote sensing. Trends Ecol Evol 18: 299-305.

Koski WR, Allen T, Ireland D, et al. 2009. Evaluation of an unmanned airborne system for monitoring marine mammals. Aquatic Mammals 35: 347-57.

Laliberte AS and Rango A. 2009. Texture and scale in object-based analysis of subdecimeter resolution unmanned aerial vehicle (UAV) imagery. IEEE T Geosci Remote 47: 761-70.

Lelong C, Burger P, Jubelin G, et al. 2008. Assessment of unmanned aerial vehicles imagery for quantitative monitoring of wheat crop in small plots. Sensors 8: 3557-85.

Lin Y. 2011. Mini-UAV-borne LiDAR for fine-scale mapping. IEEE Geosci Remote Sens 8: 426-30.

Loarie SR, Joppa LN, and Pimm SL. 2007. Satellites miss environmental priorities. Trends Ecol Evol 22: 630-32.

Mackenzie D. 2012a. A flapping of wings. Science 335: 1430-33.

Mackenzie D. 2012b. It's a bird, it's a plane, it's a...spy? Science 335: 1433 .

Mackenzie R. 2009. Environmental and Earth science using next generation aerial platforms. www.nerc.ac.uk/research/themes/ technologies/events/documents/uav-study-report.pdf. Viewed 16 May 2012.

Martin S, Bange J, and Beyrich F. 2011. Meteorological profiling of the lower troposphere using the research UAV "Mㄹ AV Carolo". Atmos Measuring Tech 4: 705-16.

Mayer S, Sandvik A, Jonassen MO, et al. 2012. Atmospheric profiling with the UAS SUMO: a new perspective for the evaluation of fine-scale atmospheric models. Meteorol Atmos Phys 116: 15-26.

Merino L, Caballero F, Martinez-de-Dios JR, et al. 2012. An unmanned aircraft system for automatic forest fire monitoring and measurement. J Intell Robot Syst 65: 533-48.

Merz T and Chapman S. 2011. Autonomous unmanned helicopter system for remote sensing missions in unknown environments. www.isprs.org/proceedings/XXXVIII/1-C22/papers/merz neu.pdf. Viewed 20 Apr 2012.
Migliavacca M, Galvagno M, Cremonese E, et al. 2011. Using digital repeat photography and eddy covariance data to model grassland phenology and photosynthetic $\mathrm{CO}_{2}$ uptake. Agric Forest Meteorol 151: 1325-37.

NOAA (National Oceanic and Atmospheric Administration). 2012. State of the science fact sheet: emerging technologies for mobile Earth observations. www.nrc.noaa.gov/plans_docs/ SoS_AandR_Platforms_FINAL.pdf. Viewed 1 Mar 2012.

Orlowski CT and Girard AR. 2012. Dynamics, stability, and control analyses of flapping wing micro-air vehicles. Prog Aerosp Sci 51: 18-30.

Rosnell T and Honkavaara E. 2012. Point cloud generation from aerial image data acquired by a quadrocopter type micro unmanned aerial vehicle and a digital still camera. Sensors 12: 453-80.

Running SW, Nemani RR, Heinsch FA, et al. 2004. A continuous satellite-derived measure of global terrestrial primary production. BioScience 54: 547-60.

Sarda-Palomera F, Bota G, Vinolo C, et al. 2012. Fine-scale bird monitoring from light unmanned aircraft systems. Ibis 154: $177-83$.

Smith GM and Milton EJ. 1999. Technical note: the use of the empirical line method to calibrate remotely sensed data to reflectance. Int J Remote Sens 20: 2653-62.

van den Kroonenberg A, Martin S, Beyrich F, et al. 2012. Spatiallyaveraged temperature structure parameter over a heterogeneous surface measured by an unmanned aerial vehicle. BoundLay Meteorol 142: 55-77.

Vierling KT, Vierling LA, Gould WA, et al. 2008. LiDAR: shedding new light on habitat characterization and modeling. Front Ecol Environ 6: 90-98.

Wallace LO, Lucieer A, Turner D, et al. 2011. Error assessment and mitigation for hyper-temporal UAV-borne LiDAR surveys of forest inventory. www.terraluma.net/downloads/Wallace_Silvi Laser2011.pdf. Viewed 12 May 2012.

Wallace L, Lucieer A, Watson C, et al. 2012. Development of a UAV-LiDAR system with application to forest inventory. Remote Sens 4: 1519-43.

Watts AC, Ambrosia VC, and Hinkley EA. 2012. Unmanned aircraft systems in remote sensing and scientific research: classification and considerations of use. Remote Sens 4: 1671-92.

Wulder MA, Hall RJ, Coops NC, et al. 2004. High spatial resolution remotely sensed data for ecosystem characterization. BioScience 54: 511-21.

Zarco-Tejada PJ, Gonzalez-Dugo V, and Berni JAJ. 2012. Fluorescence, temperature and narrow-band indices acquired from a UAV platform for water stress detection using a microhyperspectral imager and a thermal camera. Remote Sens Environ 117: 322-37. 